\title{
Separation and identification of polar polyphenols in oily formulation using high-performance thin-layer chromatography and mass spectroscopy techniques
}

\author{
Ophélie Fadel $^{1}$, Donatien Gomes Rodrigues ${ }^{1}$, Luc Girard ${ }^{1}$, Pierre Bauduin ${ }^{1}$, Anne Rossignol-Castera ${ }^{2}$, \\ Annabelle L'Hermitte ${ }^{2}$, Jean-Charles Gaillard ${ }^{3}$ and Olivier Diat ${ }^{1, *}$ \\ 1 ICSM, CEA, CNRS, ENSCM, Univ Montpellier, Marcoule, France \\ 2 OLEOS/Hallstar 50 rue du Rajol, Fréjorgues Est, 34130 Mauguio, France \\ ${ }^{3}$ CEA, Direct Rech Fondamentale IBITECS, Site Marcoule, 30207 Bagnols Sur Ceze, France
}

Received 4 July 2018 - Accepted 18 July 2018

\begin{abstract}
In cosmetics, there is some interest to solubilized polar antioxidant molecules in oil. This is a matrix that can indeed protect these fragile molecules against oxidation and that makes possible the polar antioxidants transfer through the epidermis in order to be delivered within the dermis. Varying the oil formulation allows the achievement of a high content of antioxidants in a natural vegetable oil using an oleoeco extraction process. However, it was necessary to characterize this content using quantitative and efficient methods such as High-Performance Thin-Layer Chromatography technique. The aim of this work was to develop a method of antioxidant's characterization with this technique without a preliminary reextraction of the antioxidants.
\end{abstract}

Keywords: solubilisation / separation / antioxidant / oily matrices / High-performance Thin-layer Chromatography

\begin{abstract}
Résumé - Séparation et identification de polyphénols polaires dans une formulation huileuse, au moyen de techniques de chromatographie sur couche mince haute performance et de spectroscopie de masse. Il y a un intérêt d'utiliser en cosmétique des molécules antioxydantes polaires solubilisées dans une matrice huileuse. C'est une matrice qui peut en effet protéger ces molécules fragiles contre l'oxydation et qui peut favoriser le transfert d'antioxydants polaires à travers l'épiderme pour être délivrés dans le derme. Dans un procédé d'oléo-éco extraction utilisant une huile ou une cire végétale, une formulation appropriée de la matrice huileuse peut permettre d'atteindre une teneur élevée en antioxydants polaire. Cependant, il est nécessaire de caractériser ce contenu en utilisant des méthodes quantitatives et efficaces, telles que la technique de chromatographie en couche mince à haute performance afin d'optimiser le procédé. Le but de ce travail est de développer une méthode de caractérisation de l'antioxydant avec cette technique sans ré-extraction préalable des antioxydants.
\end{abstract}

Mots clés : solubilisation / séparation / antioxydant / matrices huileuses / chromatographie sur couche mince haute performance

\section{Introduction}

Nowadays, the integration of antioxidants in cosmetic, food and pharmaceutical products is a standard procedure to counterbalance oxidative stress generated in our everyday life (Bezerra et al., 2017; Valko et al., 2007). Polyphenols from

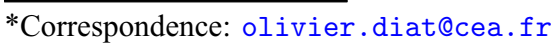

plants belong to this category of molecules (Balasundram et al., 2006). Indeed, stored in the vacuole of many plants, they are recognized as having health benefits in particular against aging and disease (Benavente-Garcia et al., 2000; Owen et al., 2000; Rigacci and Stefani, 2016). Among the sources of phenolic compounds, olive leaves are rich in polyphenols with high antioxidant capacity (Erbay and Icier, 2010; Goulas et al., 2009; Lee et al., 2009). Pruning of the olive tree crops generates a considerable volume of biomass and thus presents 
the main source of olive leaves for the extraction of antioxidant. The oleuropein, from the secoiridoid polyphenol family, is the major constituent found in the olive-leaf extract from Olea europaea. It is a hydroxytyrosol ester with $\beta$-glucosylated elenolic acid. Others flavonoids are also present in olive leaves, either in an aglycone form (apigenin, luteolin) or in a glycosylated one (luteolin-7-O-glucoside).

Today, to preserve their potential biological activity as long as possible until their final use, polyphenols from plants have to be extracted and stored effectively following specific and green conditions (Chemat et al., 2012). Many extraction techniques have been developed. Some of them are dedicated to the process itself (Rombaut et al., 2014), using for example microwaves or ultrasounds (Chemat et al., 2017; Japon-Lujan and de Castro, 2006; Pan et al., 2003), some others are based on the use of alternative green solvents (Filly et al., 2015; Gu and Jerome, 2013; Haeckl and Kunz, 2018), some combine both (Achat et al., 2012). In the context of these studies, the use of vegetable oils as green and safe solvents belongs to the growing demand for using renewable resources (Li et al., 2014; Rombaut et al., 2014; Yara-Varon et al., 2017). Indeed, vegetable oils are rather complex in their composition, which makes them rather efficient as solvents for solubilizing hydrophobic and hydrophilic species. They already contain healthy molecules such as triglycerides, triterpenes, tocopherol, squalene and phenols (Owen et al., 2000; SanchezQuesada et al., 2013) and are some excellent alternative solvents to petroleum ones for integration in cosmetic, food or pharmaceutical formulations. However, they are oils, and the enhancement of hydrophilic polyphenol extraction yields within these oily matrices remains an issue. The extraction or solubilisation efficiency of hydrophilic polyphenols can vary as a function of the supramolecular structure of the oils ( $\mathrm{Li}$ et al., 2015), the polarity of polyphenols and their molecular weight (Giron et al., 2009). In a more recent work, we investigated the role of the micellisation in vegetable oils using a bio-based co-solubilizing molecule to enhance the solubility of polar antioxidants and their extraction yields (Fadel et al., 2017; Gomes Rodrigues et al., 2017). Some comparative experiments and simulations were carried out to analyze and to understand the solubilisation efficiencies. However, to achieve a more quantitative identification of the polyphenol molecules after extraction, high performance techniques such as (RP-)HPLC-DAD or (RP-)HPLC-MS (/MS) have to be used (Ajila et al., 2011; Kontogianni, 2014). However, in that peculiar case of oily systems, it requires a preliminary step of polyphenol's re-extraction (Sukumar et al., 2008), such as solvent extraction (SE) (TasioulaMargari and Tsabolatidou, 2015) or solid phase extraction (SPE) (Liberatore et al., 2001; Nevado et al., 2009; Servili et al., 1999).

The objective of this study was to find an efficient and fast chromatographic method for the qualification and quantification of polar polyphenols solubilized in vegetable oils after the plant extraction and without phase change pre-treatment. The HighPerformance Thin-Layer Chromatography (HPTLC) technique presents many advantages, including simplified sample preparation, low operating costs, short analysis time and simultaneous analysis of several samples. Thus, quantitative results from an HPTLC chromatogram analysis make it possible to determine the best extraction conditions to optimize a process.

\section{Material and methods}

\subsection{Chemical and materials}

Various solvents for LC migration such as cyclohexane, dichloromethane, ethyl acetate, methanol, phosphoric acid, acetonitrile, formic acid and ethanol were supplied by Carlo ERBA (France) in HPLC grade and without further purification. Diphenylboric acid- $\beta$-ethylamino ester or Neu reagent (Merck) was used as a derivatization agent on the HPTLC plates supplied also by Merck (Germany). Various stationary phases were investigated: HPTLC silica gel 60 F254 and HPTLC silica gel $60 \mathrm{RP}-18 \mathrm{~F} 254 \mathrm{~s}$, both on glass plates $(10 \mathrm{~cm} \times 20 \mathrm{~cm})$.

For EPR measurements, isooctane solvent was also supplied by Carlo ERBA and Galvinoxyl, used as free-radical scavenger, was supplied by Sigma-Aldrich (France) and used as received. The Folin-Ciocalteu reactive was also supplied by Sigma-Aldrich.

Ultrapure water (Milli-Q Labo, Millipore, 18 Mohm) was used in all samples preparations.

The Jojoba wax was obtained from Aroma Herbiotech (France). The amphiphilic compound, Polyglyceryl-3 Diisostearate (PG3DS) represented (CAS 66082-42-6/85666-92-8) is also a product of Gattefossé (France) with a molar mass of $773.2 \mathrm{~g} / \mathrm{Mol}$ and a density of 0.935 (Fadel et al., 2017). The Effialine $^{(1)}$ from Purextract (France) was used as a model mixture of antioxidant molecules. It contains antioxidant molecules from olive tree leaf and its composition is roughly 50 to $70 \%$ oleuropein, 1 to $5 \%$ hydroxytyrosol, 5 to $10 \%$ oleuroside and 5 to $15 \%$ luteolin glucoside. This mixture is a yellow-brown powder partially soluble in water. Oleuropein and luteolin glucoside were purchased from Sigma Aldrich and used as received. Lecithin S75 (containing $70 \mathrm{wt} . \%$ of palmitoyl-oleoyl-sn-phosphatidylcholine [POPC]) were provided by Lipoid (Grasse, France).

\subsection{Sample preparation}

The oily extracts are obtained via an Oleo-eco-extraction process (OEE) (Rossignol-Castera, 2010). The oily matrix was a jojoba wax containing $5 \mathrm{wt} \%$ of PG3DS for sample ${ }^{\circ} 1,5 \mathrm{wt} \%$ of lecithin in sample $\mathrm{n}^{\circ} 3$ and $5 \mathrm{wt} \%$ of a mixture of PG3DS and lecithin in sample $\mathrm{n}^{\circ} 2$. Starting from a mixture of cryo-grinded pieces of dried olive leaves mixed into jojoba oil above its fusion temperature and containing different fractions of amphiphilic molecules (PG3DS and/or lecithin), the samples were submitted to a series of short cycles of ultrasound $(10-30 \mathrm{kHz})$ and microwave (about $20 \mathrm{~kW} / \mathrm{kg}$ ), and under controlled atmosphere $\left(\mathrm{N}_{2}\right)$. This process makes it possible to transfer among others some of the polyphenol molecules from the leaves towards the oily phase which is filtered at the end of the process. We obtained 3 extracts numbered from 1 to 3 corresponding to the three matrices composition listed above. The objective was to identify and quantify the amount of antioxidant molecules solubilized in each of the extracts.

\subsection{Folin-Ciocalteu method}

The total phenol concentration was determined for each extract in equivalent of oleuropein concentration, following the Folin-Ciocalteu method in alkaline conditions and using a 
UV-visible detection (Mylonaki et al., 2008; Singleton et al., 1999). An oily sample solution $(0.5 \mathrm{~g})$ was first contacted with $20 \mathrm{~mL}$ of $\mathrm{MeOH} /$ water (70/30) solution to extract the phenols molecules from the oily phase. After mixing, the diphasic system was centrifuged at $6000 \mathrm{rpm}$ during $10 \mathrm{~min}$ to fully separate the methanolic phase from the oily phase. The operation was repeated twice. The methanolic phase containing the phenol molecules was then washed with $40 \mathrm{~mL} \mathrm{n}$ heptane. In a second step, $5 \mathrm{~mL}$ of distilled water containing the Folin-Ciocalteu reactive $(0.2 \mathrm{M})$ were added to $2 \mathrm{~mL}$ of the methanolic solution. The new mixture was vigorously stirred, and then $5 \mathrm{~mL}$ of a $10 \mathrm{wt} \%$ aqueous sodium carbonate solution was added, mixed and finally placed in the dark for $30 \mathrm{~min}$. The $\mathrm{UV}$-vis absorbance peak at $760 \mathrm{~nm}$ is related to the reduction of the Folin reactive by the phenols (from yellow to blue solution) and was recorded using a Jasco V-630 spectrometer. The phenol concentration [ph] in $\mathrm{ppm}$ or $\mathrm{mg} / \mathrm{kg}$ was thus given by the following equation in oleuropein equivalent:

$$
[\mathrm{ph}]_{\text {Ole eq }}=\frac{A \cdot V}{\varepsilon \cdot e \cdot f \cdot m},
$$

where $V$ is the volume of the dosed solution $\mathrm{mL}, f$ is the dilution's factor at the step 2 (6.5 for this purpose), $A$ the absorbance, $e$ the spectrometer cell thickness (in $\mathrm{cm}$ ), $m$ the mass of oily sample $(0.5 \mathrm{~g})$ and finally $\varepsilon$ the mass extinction coefficient determined using a calibration procedure with oleuropein at known concentration and following the same protocol. The titrations were performed at least in triplicate to get an experimental error of less than $\pm 10 \%$.

\subsection{Electron paramagnetic resonance (EPR) spectroscopy}

Electron paramagnetic resonance (EPR) measurements were carried out at room temperature $20^{\circ} \mathrm{C}$, using a Bruker EMX EPR spectrometer operating at X-band. Samples were contained in a high purity quartz cell. Typical instrument settings were the center field at $3505 \mathrm{G}$, the scan range $100 \mathrm{G}$, the sweep time $5 \mathrm{~s}$, the receiver gain $30 \mathrm{~dB}$, the modulation amplitude $1 \mathrm{G}$, the modulation sequence $100 \mathrm{kHz}$, the scan number, 3 , the attenuation, $25 \mathrm{~dB}$, the power $0.66 \mathrm{~mW}$ and the frequency $9.85 \mathrm{GHz}$. Data collection and analysis were performed using Xenon software (acquisition and processing program).

Samples containing the phenolic compounds were examined for their radical scavenging activity toward the stable galvinoxyl radical (Galv-O) by EPR spectroscopy (Papadimitriou et al., 2006). A $0.15 \mathrm{mM}$ solution of Galv-O in isooctane was analyzed for reference before adding $2 \% \mathrm{v} / \mathrm{v}$ of the samples. Signal intensity of Galv-O in isooctane consists of a broad peak which decreases in intensity upon samples addition. The signal decrease, $\mathrm{D}$ in percent, was calculated from the following expression:

$$
\mathrm{D}=\left(\mathrm{I}_{0}-\mathrm{I}\right) / \mathrm{I}_{0} \times 100,
$$

where $I_{0}$ is the intensity of the EPR spectrum of galvinoxyl solution and $I$ is the intensity of the EPR spectrum in the presence of the oil sample. A parallel control using a system containing the same volume of isooctane in the galvinoxyl solution was performed in order to check the stability of the signal.

\subsection{High-performance thin-layer chromatography}

\subsubsection{HPTLC method}

The samples were sprayed as $8 \times 4 \mathrm{~mm}$ spots on one HPTLC plate of $20 \times 10 \mathrm{~cm}$ (distance between spots :12.4, 17.4 or $22.4 \mathrm{~mm}$; distance from the lower edge: 9 and $10 \mathrm{~mm}$; distance from the left side: 19.4, 20.4 or $21.6 \mathrm{~mm}$ ) using an Automatic TLC Sampler 4 (ATS4). Sample volumes ranged between 1 and $20 \mu \mathrm{l}$. The HPTLC plates were developed using different eluents in a pre-saturated ADC2 chamber (Camag; Muttenz, Switzerland). The conditions of development were $20 \mathrm{~min}$ for the tank saturation time, $22.5^{\circ} \mathrm{C}$ for temperature of operation, 40 and $60 \% \mathrm{RH}$ for the humidity state, 60 and $85 \mathrm{~mm}$ for the migration distance, and $5 \mathrm{~min}$ for the plate drying time. The plate's derivatization was carried out by immersion in a solution of Neu reagent $(1 \mathrm{~g}$ of diphenyl boric acid ethylamino ether in $200 \mathrm{~mL}$ of ethyl acetate) using an immersion device (Camag; Muttenz, Switzerland) after the plate's heating at $100^{\circ} \mathrm{C}$ for 3 min on a TLC plate heater (Camag; Muttenz, Switzerland). Finally, the HPTLC plates were photographed by a TLC Visualizer (Camag; Muttenz, Switzerland).

\subsubsection{Reversed phase system}

After a pre-migration with cyclohexane up to $85 \mathrm{~mm}$, a development was performed with a mixture of cyclohexaneethyl acetate-methanol-phosphoric acid (58:24:18:0.5; v/v/v/ v) to $60 \mathrm{~mm}$. Developed plates were photographed before and after derivatization.

\subsubsection{Normal phase system}

After a pre-migration with cyclohexane or dichloromethane up to $85 \mathrm{~mm}$, development was performed with first either a similar mixture of cyclohexane-ethyl acetate-methanol-phosphoric acid but at two different proportions $(58: 24: 18: 0.5$ or $30: 30: 20: 0.4 ; \mathrm{v} / \mathrm{v} / \mathrm{v} / \mathrm{v})$ or with a mixture of dichloromethaneacetonitrile- formic acid-water $(40: 10: 1.6: 0.6 ; \mathrm{v} / \mathrm{v} / \mathrm{v} / \mathrm{v})$ up to $60 \mathrm{~mm}$.

\subsubsection{Description of optimized chromatographic settings}

These HPTLC characterization being fully repeatable, a general and optimized chromatographic setting can be described as follows: a first solubilisation of the sample is performed in cyclohexane/dichloromethane $(1: 1: 1)$. The size of the sample stains on the solid substrate is about $8 \times 4 \mathrm{~mm}^{2}$, corresponding to a dropped volume of less than $20 \mu \mathrm{l}$. We used as silica gel stationary phase either RP-18 or $60 \mathrm{~F} 254$ plates. The total migration front was $85 \mathrm{~mm}$. The fluorescence development was carried out using Neu reagent with an immersion speed of $3 \mathrm{~s}$, an immersion lap of $1 \mathrm{~s}$ and a heating temperature of $100{ }^{\circ} \mathrm{C}$ for $3 \mathrm{mn}$.

\subsubsection{Mass spectrometry}

HPTLC-ESI-MS spectra were directly recorded using the TLC-MS Interface (CAMAG) equipped with the oval elution head $(2 \times 4 \mathrm{~mm})$. It was coupled with the electrospray ionization (ESI) interface of LTQ Orbitrap XL hybrid mass spectrometer (ThermoFisher Scientific). The respective zone was directly eluted with an aqueous solution of $90 \%$ of $\mathrm{CH}_{3} \mathrm{OH}$ 


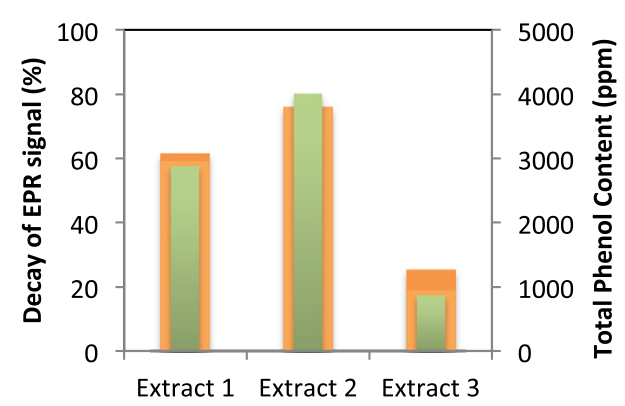

Fig. 1. Antioxidant activity (orange) and Total Phenol Content (green) of three extracts obtained by oleo-eco-extraction.

and $0.1 \%$ formic acid at a flow rate of $200 \mu \mathrm{l} / \mathrm{min}$ (LoadingPump of NCS-3500RS ThermoFisher Scientific). The LTQ Orbitrap XL instrument worked under the following conditions: in normal mode full scan with linear ion trap, the mass range was $\mathrm{m} / \mathrm{z} 150-1400$ with a positive ion detection mode, the ESI voltage was set to $3800 \mathrm{~V}$, the temperature of the heated capillary was $300^{\circ} \mathrm{C}$, the capillary voltage set to $49 \mathrm{~V}$, and the tube lens voltage to $125 \mathrm{~V}$. Other LTQ Orbitrap XL parameters were automatically optimized by the system.

\section{Results and discussion}

\subsection{Non-specific antioxidant titration}

The first step towards characterizing the extraction efficiency of the OEE process was to determine the total phenol content and the antioxidant activity in each extract because antioxidant capacity of olive leaves depends on the phenolic profile (Laguerre et al., 2009). We used two different methods. The Folin method for the former and the free radical trap via EPR technique for the latter. Both results for each of the three extracts are plotted in Figure 1 in orange and green for the antioxidant activity and the total phenol content in oleuropein equivalent, respectively. On Figure 1, we can observe that the anti-oxidant activity and the polyphenol content variation are correlated, although the proportionality is not followed. Indeed, the EPR technique makes it possible to evaluate the capacity of polyphenols solubilized in the oil to trap the free radicals, whereas the Folin method makes it possible to titrate the amount of polyphenol in a non-specific way. Nevertheless, the results show that the efficiency of polyphenols extraction from the same olive leaves source depends on the oily matrix. Indeed, depending on the oily matrix, the amount of polyphenol titrated varied from a factor 1 to 3 . However, the non-specificity of both methods does not make it possible to optimize the conditions of the process in order to extract more specifically a molecule with a strong interest for either a cosmetic or a pharmacologic application.

Thin layer chromatography is now a common method to separate, on a stationary phase) via the migration of a specific mobile phase (Loescher et al., 2014; Nicoletti, 2011). Vegetable oils and OEE extracts are complex mixtures that contain apolar, polar and sometimes amphiphilic molecules that are difficult to separate using the same eluents or mixture of eluents. Often, pre-separation or pre-extraction are mandatory to decrease the number of components.
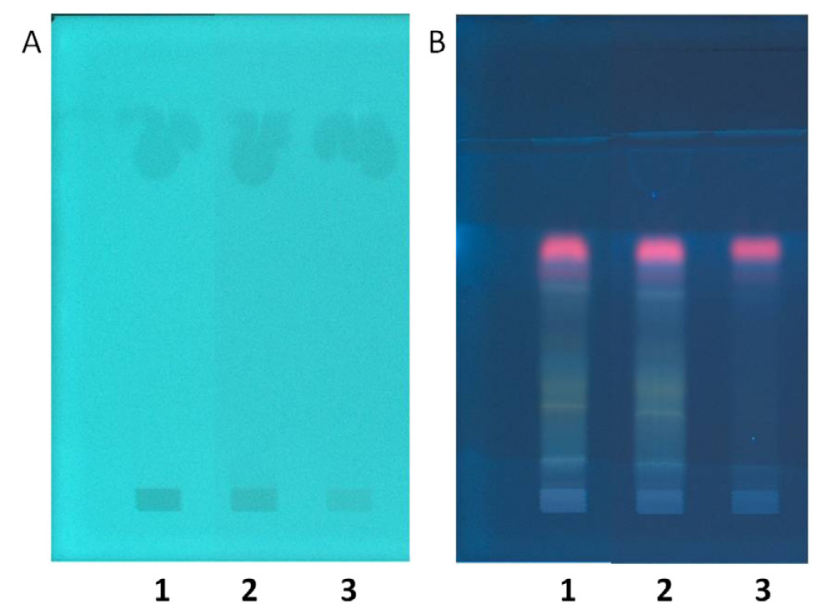

Fig. 2. HPTLC fingerprints of three extracts n.1, 2 and 3 on plates RP-18: A) After a pre-migration step with cyclohexane as the mobile phase and illuminated under white light; B) development in a second step with cyclohexane-ethyl acetate-methanol-phosphoric acid 58:24:18:0.5, illuminated under $366 \mathrm{~nm}$.

In this section, we will compare chromatographic methods on apolar and polar silica plates to show that with a suitable migration in an appropriate mobile phase/stationary phase couple, it is possible to avoid preliminary extraction steps such as SPE that can require large volumes of sample and then directly drop-off the oily samples on the plate for analysis.

\subsection{TLC on apolar stationary phase}

After the drop-off of the extracts on the apolar or reversed phase silica plate, a first migration was performed using cyclohexane, one of the least polar solvent. This first step yields a first migration of the apolar components such as the mono-esters of long-chain fatty acids, the main component of jojoba oil, at the solvent front, at the top of the plate (see Fig. 2A). They are distributed in a relatively large stain with a flower shape. However, we can clearly observe some chemicals that remained in the extracts visible under the white light. This tells us that these components are much more polar than the mono-ester molecules. A second migration with cyclohexane-ethyl acetate-methanol-phosphoric acid 58:24:18:0.5 was performed and now several traces with different colors were developed at various retarding factors (see Fig. 2B). Some of them are rather thick and intense like the pink traces that correspond to the chlorophylls (Havlikova et al., 2014). Some others are rather thin and well defined and appear either blue or yellow when illuminated at $366 \mathrm{~nm}$. Finally, we can also observe some very fuzzy bands which we did not succeed in intensifying by changing the composition of the migration solvent.

Nevertheless, the bands are parallel and visible for the three extracts only with different intensities that are correlated with the previous determination of the polyphenol content and antioxidant activity.

\subsection{TLC on polar stationary phase}

A similar two-step migration was performed for the three extracts now using a polar silica gel 60 F254. The first step 
using cyclohexane as the first eluent shows in white light (see Fig. 3A) some long and spread tears. The second step using the cyclohexane-ethyl acetate-methanol-phosphoric acid mixture used previously reveals a different series of bands that appear blue, far from the eluent front at low $\mathrm{R}_{\mathrm{f}}$ and pink near the eluent front at high $\mathrm{R}_{\mathrm{f}}$. These bands differ chemically from those separated using the apolar silica stationary phase, are slightly better defined but remain relatively weak in intensity. However, as previously mentioned, the band series for the extract $n .2$ looks slightly more intense than for the two other extract signatures. Nevertheless, we can notice that the overall migration was limited by the presence of large oily stain at the eluent front that requires a different pre-migration for the step 1 .

Thus, dichloromethane was used instead of cyclohexane. As shown in Figure 4A for the three samples, and as observed
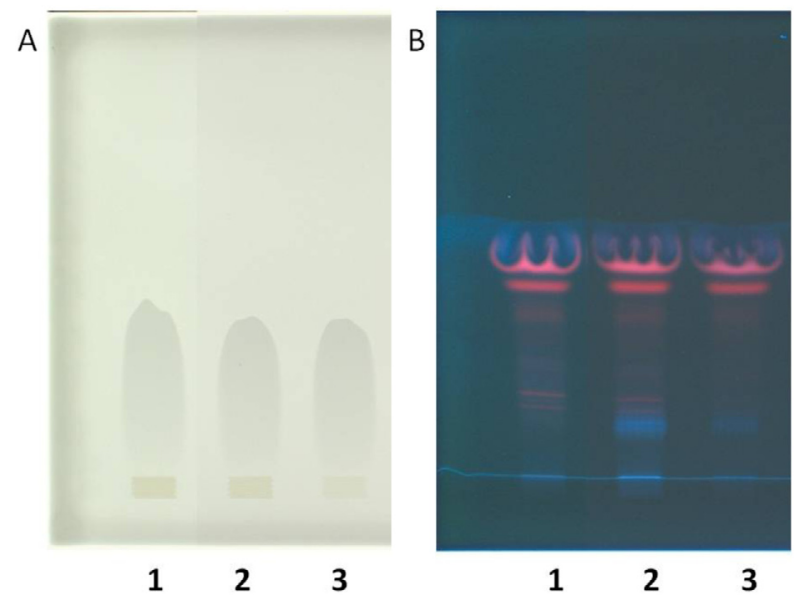

Fig. 3. HPTLC fingerprints of three extracts n.1, 2 and 3 on silica gel 60 F254 plates: A) After a pre-migration step with cyclohexane as the mobile phase and illuminated under white light; B) development in a second step with cyclohexane-ethyl acetate-methanol-phosphoric acid 58:24:18:0.5, illuminated under $366 \mathrm{~nm}$. for the first experiment on apolar silica plates, the most nonpolar components of the oil (but not highly apolar) were eluted up to the top of the plate. Dichloromethane, characterized by a solubility parameter slightly higher than those of cyclohexane, appears to be a more suitable mobile phase for this primary migration. Thus, during the second migration step and using a mixture of cyclohexane-ethyl acetate-methanol-phosphoric acid 58:24:18:0.5, the separation of the secondary compounds (compounds other than mono-esters) appears much more distributed along the fingerprints (see Fig. 4B). The fluorescence of the different tracks under UV, especially those that appear between the sample's stain and the front of the second eluent where the chlorophyllic compounds are revealed, is enhanced via the Neu post-chromatographic treatment as shown in Figure 4C.

In order to further optimize the separation of the different species, the second step was carried out using a dichloromethane-acetonitrile-formic acid-water 40:10:1.6:0.6 mixture. This type of mixture was already used for secoiridoides separation like the oleuropeine (Czerwinska et al., 2015), a polar compound that is expected to be encountered in our extracts. The silica plate was then illuminated under UV-366 nm and we observed a much better separation of the various polar antioxidant molecules on the lower part of the chromatographic plate, characterized by a lower $\mathrm{R}_{\mathrm{f}}$ (see Fig. 5, left-hand side).

Once the chromatographic separation was optimized, references were prepared and submitted to the last TLC protocol used. Eff track corresponds to a sample containing the Effialine solubilized in ethanol. A chlorophyll reference was also used (track Chl) as well as an ethanoic solution of luteolin7-glucoside (track Lut) and an ethanoic solution of oleuropein (track Ole) (see Fig. 5). The comparison of the different tracks obtained in the same chromatographic conditions confirms first the presence and the separation of the chlorophyll compounds for the three extracts that appear in pink colours and transferred in the upper part of the plate. It also highlights the presence of polyphenols for the three extracts in agreement with the first results concerning the antioxidant activities. However, it appears clearly that the three extracts contain different fractions of these molecules and thus depends on the OEE
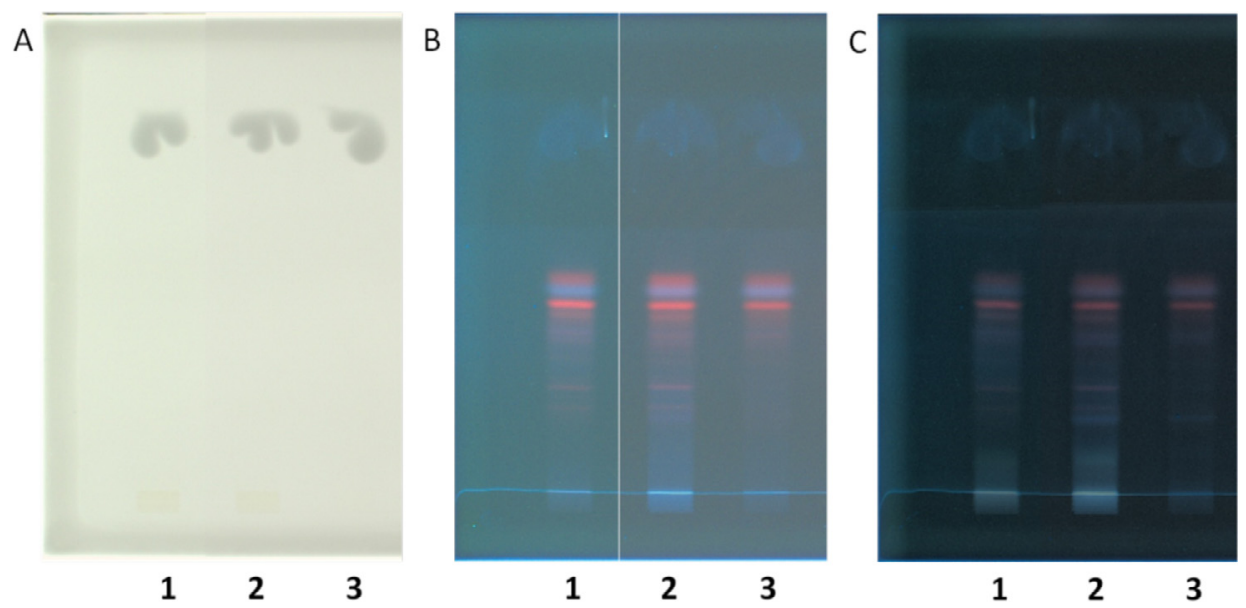

Fig. 4. HPTLC fingerprints of three extracts n.1, 2, 3 on silica gel 60 F254 plates: A) After a pre-migration step with dichloromethane as the mobile phase and illuminated under white light; B) development in a second step with cyclohexane-ethyl acetate-methanol-phosphoric acid 50:30:20:0.4, illuminated under $366 \mathrm{~nm}$ before derivatization; C) after derivatization with Neu reagent. 
formulation. For the extract n.3, very small amounts of polyphenols are extracted and mainly chlorophyll is present. On the other hand, polyphenol molecules have been extracted in a larger amount in the oily extracts n.1 and n.2. Oleuropein, the major compound of the olive leaves and luteolin-7glucoside can be detected with a ratio different from what exists in the Effialine. It is important to notice that along the migration strip of the Oleuropein, the compound does not appear really pure with the presence of several blue bands. However, the most intense and resolved one is those located at an $\mathrm{Rf}$ of 0.46 and that is detected within the fingerprints of the three extracts.

\subsection{TLC-(ESI)MS}

In complexed oily extracts, it remains difficult to confirm the solubilized molecules based only on the comparisons between HPTLC fingerprints obtained from

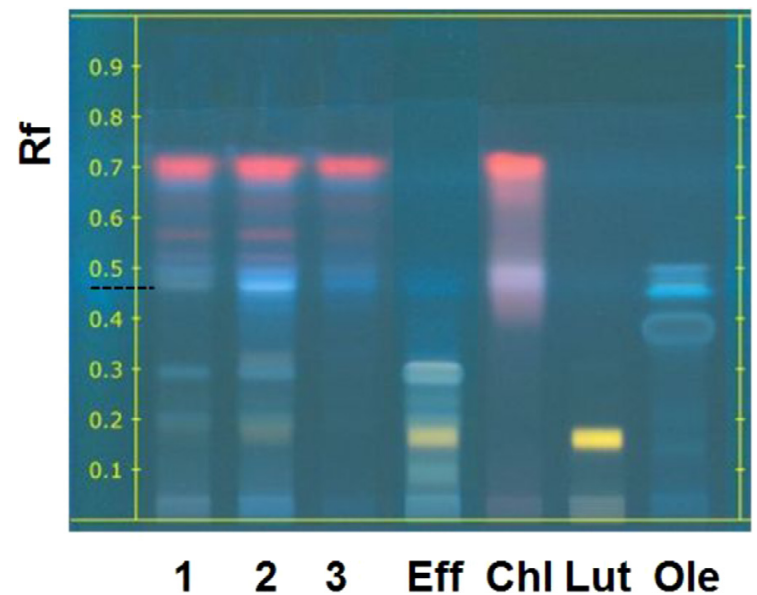

Fig. 5. HPTLC fingerprints of three extracts n.1, 2 and 3 as well as for four referenced samples, Effialine (Eff), chlorophyll (Chl), uteolin-7glucoside (Lut) and oleuropein (Ole), on silica gel 60 F254 plates after a pre-migration step with dichloromethane and with dichloromethane-acetonitrile- formic acid-water 40:10:1.6:0.6 in a second step, illuminated under $366 \mathrm{~nm}$ after derivatization. referenced samples. However, owing to the coupling with mass-spectroscopy using a specific interface, a more appropriate identification is possible and needed. From previous observations, the intense blue band at $36 \mathrm{~mm}$ from the initial droplet $\left(\mathrm{R}_{\mathrm{f}}=0.46\right)$ of the extract $n .2$ that appears to contain the oleuropein as well as the band at the same level from the Effialine reference were eluted with mixture of methanol and formic acid via the HPTLC/MS interface and sent to the MS. The oleuropein signature with the right $\mathrm{m} / \mathrm{z}\left([\mathrm{M}+\mathrm{Na}]^{+} \sim 563.2\right)$ was identified in both cases (see Fig. 6A and B) and agreed with results found in literature (Mylonaki et al., 2008; Obied et al., 2007). However, this detection was not reproduced with extract $n .1$ and $n .2$ due to a lower concentration on the plates. Moreover, a second peak at higher mass was detected for extract $n .2$ for $\mathrm{m} / \mathrm{z}$ ( 677.6). It was not possible to assign corresponding species or dimers present in the oily matrix and further analyses are required to qualify it. It should correspond to a slightly more apolar compound and exists because the area probed via the HPTLC/MS interface is too large and can cover two existing bands when they are not sufficiently separated through the solvent migration and as observed in Figure 5 at $\mathrm{R}_{\mathrm{f}}=0.46 \pm 0.03$.

\section{Conclusions}

The major component of the polyphenols found in olive leaves, the oleuropein, has been separated and identified among different oily extracts obtained via an oleo-ecoextraction process and without using any pre-treatment such as SE or SPE type methods. We have only exploited an optimized dilution method using high performance thin layer chromatography technique coupled to ESI-Mass spectrometer.

The advantage of the HPTLC technique is here fully retained with a quick and simultaneous analysis and identification of various apolar and polar compounds dispersed in an oily matrix, using referenced systems and small amount of samples.

Concerning the samples that we have studied, we have shown that the formulation $n .2$ containing a mixture of PG3DS and lecithin in a jojoba wax matrix is a most efficient mixture to extract some specific antioxidant molecules present in olive

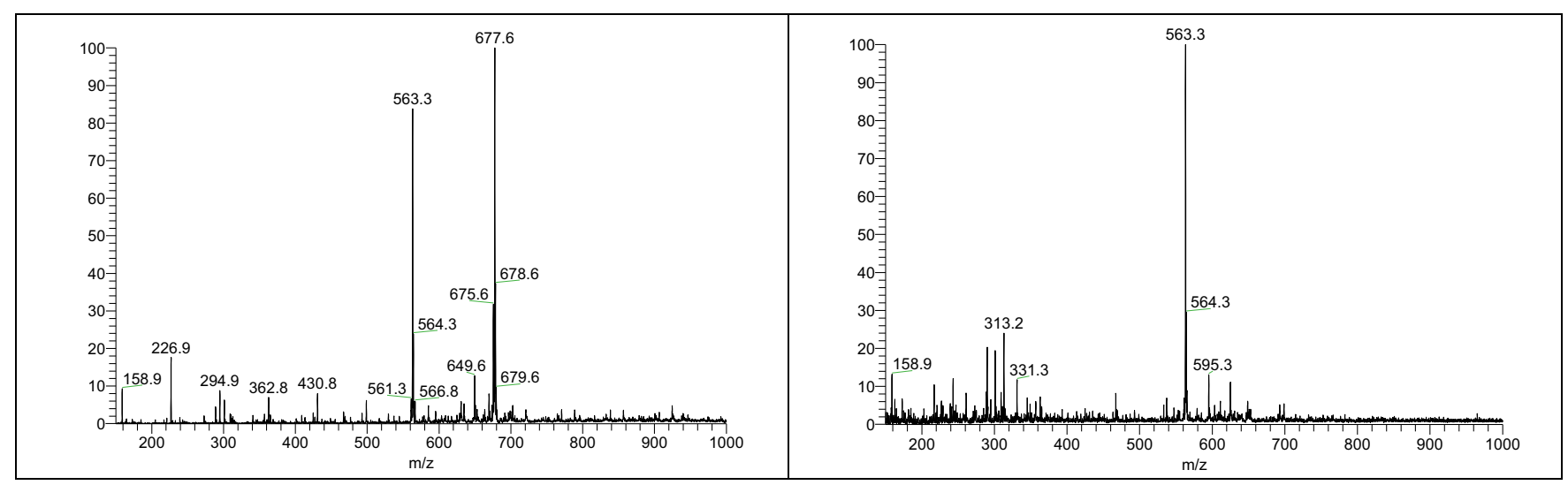

Fig. 6. Characterization of Oleuropein in extract 2 (left) and Effialine (right) by HPTLC-ESI-MS Oleuropein expected at m/z 539.2, observed with a $\mathrm{Na}$ adduct at $\mathrm{m} / \mathrm{z} 562.3:(539.2+23 \mathrm{Na}) \pm 0.1$ Atomic mass of Oleuropein is $540.2 \mathrm{u}$. 
leaves. In this study, the oleuropein is the predominant species among the extracted polyphenols. A more quantitative analysis requires a fine development of the coupling between HPTLC and MS in optimizing the band selection along the migration strip. Although each band was well defined, the optimization of the eluent's formulation to extract the species from the silica plates is necessary to allow for the identification of a very small amount of separated polyphenols and perhaps some other chemical compounds extracted during the OEE process. We have demonstrated that this method can allow for a quick evaluation of the extraction yield and a selection of the best OEE process parameters.

Acknowledgements. This work was supported by the National funding ANR-14-LAB4-0005-01 (Vect'Oleo). The authors thank Dr. X. Le Goff for his technical help in EPR settings as well as the engineers and technicians from Oleos/Hallstar who help us in running the anti-oxidant OEE processes.

\section{References}

Achat S, Tomao V, Madani K, et al. 2012. Direct enrichment of olive oil in oleuropein by ultrasound-assisted maceration at laboratory and pilot plant scale. Ultrason. Sonochem. 19: 777-786.

Ajila CM, Brar SK, Verma M, Tyagi RD, Godbout S, Valero JR. 2011. Extraction and Analysis of Polyphenols: Recent trends. Crit. Rev. Biotechnol. 31: 227-249.

Balasundram N, Sundram K, Samman S. 2006. Phenolic compounds in plants and agri-industrial by-products: Antioxidant activity, occurrence, and potential uses. Food Chem. 99: 191-203.

Benavente-Garcia O, Castillo J, Lorente J, Ortuno A, Del Rio JA. 2000. Antioxidant activity of phenolics extracted from Olea europaea L-leaves. Food Chem. 68: 457-462.

Bezerra DP, Militao GCG, de Morais MC, de Sousa DP. 2017. The dual antioxidant/prooxidant effect of Eugenol and its action in cancer development and treatment. Nutrients 9.

Chemat F, Rombaut N, Sicaire AG, Meullemiestre A, Fabiano-Tixier AS, Abert-Vian M. 2017. Ultrasound assisted extraction of food and natural products. Mechanisms, techniques, combinations, protocols and applications. A review. Ultrason. Sonochem. 34: 540-560.

Chemat F, Vian MA, Cravotto G. 2012. Green extraction of natural products: concept and principles. Int. J. Mol. Sci. 13: 8615-8627.

Czerwinska ME, Ziarek M, Bazylko A, Osinska E, Kiss AK. 2015. Quantitative Determination of Secoiridoids and Phenylpropanoids in Different Extracts of Ligustrum Vulgare L. Leaves by a Validated HPTLC-Photodensitometry Method. Phytochem. Anal. 26: 253-260.

Erbay Z, Icier F. 2010. The importance and potential uses of olive leaves. Food Rev. Int. 26: 319-334.

Fadel O, Girard L, Gomes Rodrigues D, et al. 2017. Micellization in vegetable oils: A structural characterisation. Colloids Surf. BBiointerfaces 154: 279-286.

Filly A, Fabiano-Tixier AS, Fernandez X, Chemat F. 2015. Alternative solvents for extraction of food aromas. Experimental and COSMO-RS study. Lwt-Food Sci. Technol. 61: 33-40.

Giron MV, Ruiz-Jimenez J, de Castro MDL. 2009. Dependence of Fatty-Acid Composition of Edible Oils on Their Enrichment in Olive Phenols. J. Agric. Food Chem. 57: 2797-2802.

Gomes Rodrigues D, Fadel O, Bauduin P, et al. 2017. Self-assembly of a bio-based extractant in methyl esters: combination of small angle X-ray scattering experiments and molecular dynamics simulations. Green Chem. 19: 4680-4689.

Goulas V, Exarchou V, Troganis AN, et al. 2009. Phytochemicals in olive-leaf extracts and their antiproliferative activity against cancer and endothelial cells. Mol. Nutr. Food Res. 53: 600-608.

Gu YL, Jerome F. 2013. Bio-based solvents: an emerging generation of fluids for the design of eco-efficient processes in catalysis and organic chemistry. Chem. Soc. Rev. 42: 9550-9570.

Haeckl K, Kunz W. 2018. Some aspects of green solvents. C. r. Chim. DOI: 10.1016/j.crci.2018.03.010.

Havlikova L, Satinsky D, Opletal L, Solich P. 2014. A Fast Determination of Chlorophylls in Barley Grass Juice Powder Using HPLC Fused-Core Column Technology and HPTLC. Food Anal. Methods 7: 629-635.

Japon-Lujan R, de Castro L. 2006. Superheated liquid extraction of oleuropein and related biophenols from olive leaves. $J$. Chromatogr. A 1136: 185-191.

Kontogianni VG. 2014. Towards the identification of different classes of polyphenols. Polyphenols in Plants. Isolation, Purification and Extract Preparation. 159-176.

Laguerre M, Giraldo LJL, Piombo G, et al. 2009. Characterization of Olive-Leaf Phenolics by ESI-MS and Evaluation of their Antioxidant Capacities by the CAT Assay. J. Am. Oil Chem. Soc. 86: 1215-1225.

Lee OH, Lee BY, Lee J, et al. 2009. Assessment of phenolics-enriched extract and fractions of olive leaves and their antioxidant activities. Bioresour. Technol. 100: 6107-6113.

Li Y, Fabiano-Tixier AS, Ginies C, Chemat F. 2014. Direct green extraction of volatile aroma compounds using vegetable oils as solvents: Theoretical and experimental solubility study. Lwt-Food Sci. Technol. 59: 724-731.

Li Yu K, Fabiano-Tixier AS, Ruiz K, et al. 2015. Comprehension of direct extraction of hydrophilic antioxidants using vegetable oils by polar paradox theory and small angle X-ray scattering analysis. Food Chem. 173: 873-880.

Liberatore L, Procida G, d'Alessandro N, Cichelli A. 2001. Solidphase extraction and gas chromatographic analysis of phenolic compounds in virgin olive oil. Food Chem. 73: 119-124.

Loescher CM, Morton DW, Razic S, Agatonovic-Kustrin S. 2014. High performance thin layer chromatography (HPTLC) and high performance liquid chromatography (HPLC) for the qualitative and quantitative analysis of Calendula officinalis-Advantages and limitations. J. Pharm. Biomed. Anal. 98: 52-59.

Mylonaki S, Kiassos E, Makris DP, Kefalas P. 2008. Optimisation of the extraction of olive (Olea europaea) leaf phenolics using water/ ethanol-based solvent systems and response surface methodology. Anal. Bioanal. Chem. 392: 977-985.

Nevado JJB, Penalvo GC, Robledo VR, Martinez GV. 2009. New CEESI-MS analytical method for the separation, identification and quantification of seven phenolic acids including three isomer compounds in virgin olive oil. Talanta 79: 1238-1246.

Nicoletti M. 2011. HPTLC fingerprint: a modern approach for the analytical determination of Botanicals. Rev. Bras. Farmacogn. 21: 818-823.

Obied HK, Bedgood DR, Prenzler PD, Robards K. 2007. Chemical screening of olive biophenol extracts by hyphenated liquid chromatography. Anal. Chim. Acta. 603: 176-189.

Owen RW, Mier W, Giacosa A, Hull WE, Spiegelhalder B, Bartsch H. 2000. Phenolic compounds and squalene in olive oils: the concentration and antioxidant potential of total phenols, simple phenols, secoiridoids, lignans and squalene. Food Chem. Toxicol. 38: 647-659. 
Pan XJ, Niu GG, Liu HZ. 2003. Microwave-assisted extraction of tea polyphenols and tea caffeine from green tea leaves. Chem. Eng. Process. 42: 129-133.

Papadimitriou V, Sotiroudis TG, Xenakis A, Sofikiti N, Stavyiannoudaki V, Chaniotakis NA. 2006. Oxidative stability and radical scavenging activity of extra virgin olive oils: An electron paramagnetic resonance spectroscopy study. Anal. Chim. Acta. 573: 453-458.

Rigacci S, Stefani M. 2016. Nutraceutical properties of olive oil polyphenols. an itinerary from cultured cells through animal models to humans. Int. J. Mol. Sci. 17.

Rombaut N, Tixier AS, Bily A, Chemat F. 2014. Green extraction processes of natural products as tools for biorefinery. Biofuels Bioprod. Biorefining-Biofpr 8: 530-544.

Rossignol-Castera A. 2010. Method for extracting non-volatile compound. WO2010112760 A1.

Sanchez-Quesada C, Lopez-Biedma A, Warleta F, Campos M, Beltran G, Gaforio JJ. 2013. Bioactive properties of the main triterpenes found in olives, virgin olive oil, and leaves of olea europaea. J. Agric. Food Chem. 61: 12173-12182.

Servili M, Baldioli M, Selvaggini R, Miniati E, Macchioni A, Montedoro G. 1999. High-performance liquid chromatography evaluation of phenols in olive fruit, virgin olive oil, vegetation waters, and pomace and 1D-and 2D-nuclear magnetic resonance characterization. J. Am. Oil Chem. Soc. 76: 873-882.

Singleton VL, Orthofer R, Lamuela-Raventos RM. 1999. Analysis of total phenols and other oxidation substrates and antioxidants by means of Folin-Ciocalteu reagent. Oxid. Antioxid. Pt. A 299: $152-178$.

Sukumar D, Arimboor R, Arumughan C. 2008. HPTLC fingerprinting and quantification of lignans as markers in sesame oil and its polyherbal formulations. J. Pharma. Biomed. Anal. 47: 795-801.

Tasioula-Margari M, Tsabolatidou E. 2015. Extraction, Separation, and Identification of Phenolic Compounds in Virgin Olive Oil by HPLC-DAD and HPLC-MS. Antioxidants 4: 548-562.

Valko M, Leibfritz D, Moncol J, Cronin MTD, Mazur M, Telser J. 2007. Free radicals and antioxidants in normal physiological functions and human disease. Int. J. Biochem. Cell Biol. 39: 44-84.

Yara-Varon E, Li Y, Balcells M, Canela-Garayoa R, Fabiano-Tixier AS, Chemat F. 2017. Vegetable oils as alternative solvents for green oleo-extraction, purification and formulation of food and natural products. Molecules 22: 1474-1498.

Cite this article as: Fadel O, Gomes Rodrigues D, Girard L, Bauduin P, Rossignol-Castera A, L'Hermitte A, Gaillard J-C, Diat O. 2018. Separation and identification of polar polyphenols in oily formulation using high-performance thin-layer chromatography and mass spectroscopy techniques. OCL 25(5): D506. 